\title{
Does the use of inhaled corticosteroids in asthma benefit lung function in the long-term? A systematic review and meta-analysis
}

\author{
Daniel J. Tan ${ }^{1}$, Din S. Bui ${ }^{1}, X_{\text {in Dai }}{ }^{1}$, Caroline J. Lodge ${ }^{1}$, Adrian J. Lowe ${ }^{1}$, \\ Paul S. Thomas ${ }^{2}$, Deborah Jarvis (10 ${ }^{3}$, Michael J. Abramson (104, \\ E. Haydn Walters ${ }^{1,5}$, Jennifer L. Perret (i) ${ }^{1,6,7}$ and Shyamali C. Dharmage ${ }^{1,7}$
}

@ERSpublications

In patients with mild asthma, maintenance inhaled corticosteroids are associated with modest agedependent improvements in long-term lung function https://bit.ly/32K2ZTF

Cite this article as: Tan DJ, Bui DS, Dai X, et al. Does the use of inhaled corticosteroids in asthma benefit lung function in the long-term? A systematic review and meta-analysis. Eur Respir Rev 2021; 30: 200185 [https://doi.org/10.1183/16000617.0185-2020].

ABSTRACT While asthma is known to be associated with an increased risk of progressive lung function impairments and fixed airflow obstruction, there is ongoing debate on whether inhaled corticosteroids (ICS) modify these long-term risks.

Searches were performed of the PubMed, Embase and CENTRAL databases up to 22 July 2019 for studies with follow-up $\geqslant 1$ year that investigated the effects of maintenance ICS on changes in lung function in asthma.

Inclusion criteria were met by 13 randomised controlled trials (RCTs) (n=11678) and 11 observational studies $(\mathrm{n}=3720)$. Median (interquartile range) follow-up was 1.0 (1-4) and 8.4 (3-28) years, respectively. In the RCTs, predominantly in individuals with mild asthma, ICS use was associated with improved prebronchodilator (BD) forced expiratory volume in $1 \mathrm{~s}\left(\mathrm{FEV}_{1}\right)$ across all age groups $(2.22 \%$ predicted $(95 \%$ CI 1.32-3.12), $\mathrm{n}=8332$ ), with similar estimates of strength in association for children and adults. Improvements in post-BD $\mathrm{FEV}_{1}$ were observed in adults $(1.54 \%(0.87-2.21), \mathrm{n}=3970)$, but not in children (0.20\% (-0.49-0.90), $\mathrm{n}=3924)$ (subgroup difference, $\mathrm{p}=0.006)$. Estimates were similar between smokers and nonsmokers. There were no RCT data on incidence of fixed airflow obstruction. In the observational

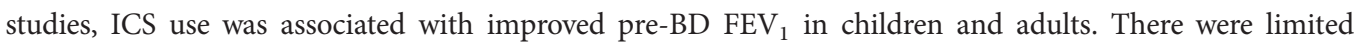
observational data for post-BD outcomes.

In patients with mild asthma, maintenance ICS are associated with modest, age-dependent improvements in long-term lung function, representing an added benefit to the broader clinical actions of ICS in asthma. There is currently insufficient evidence to determine whether treatment reduces incidence of fixed airflow obstruction in later life.

This article has supplementary material available from err.ersjournals.com

This study is registered at www.crd.york.ac.uk/prospero with identifier CRD42017053543

Provenance: Submitted article, peer reviewed.

Received: 9 June 2020 | Accepted after revision: 18 Aug 2020

Copyright $\odot$ ERS 2021. This article is open access and distributed under the terms of the Creative Commons Attribution Non-Commercial Licence 4.0. 


\section{Introduction}

Asthma is a common global disease, affecting over 350 million people, for which many patients are treated using inhaled corticosteroids (ICS). While the benefits of such treatment have been demonstrated in terms of asthma control, hospital admissions and mortality, [1-4], the long-term effects of ICS in asthma on changes in lung function remains unclear. This information is necessary to guide optimal asthma management, particularly for patients with early-onset persistent disease who are the greatest risk of progressive lung function impairments [5].

Recent studies have shown that several lung function trajectories lead to fixed airflow obstruction [6, 7]. These include impaired lung growth in the first two to three decades of life and accelerated lung function decline from the third decade onwards [6]. Depending on age of onset, asthma is now known to contribute to both pathways $[8,9]$. Given the rising prevalence and global impact of obstructive airways diseases, strategies aimed at preserving lung function in asthma are urgently needed. There is also growing international interest in this area, including a European Respiratory Society clinical initiative, CADSET (Chronic Airway Diseases Early Stratification), which aims to explore the factors that influence lung function trajectories over the lifespan [10].

ICS are currently recommended as a first-line option in persistent asthma [11], and are associated with improved airway calibre through a reduction in airway inflammation, mucus hypersecretion and bronchial hyperresponsiveness [12]. To date, the available evidence on their long-term effects on lung function in asthma have not been adequately summarised. Updates to the Global Initiative for Asthma (GINA) recommendations in 2019 [13], which now strongly advocate for ICS-containing treatment in all patients with mild asthma, further increase the need to critically evaluate the evidence on this topic. Therefore, we aimed to systematically review all studies which have investigated the long-term effects of ICS on lung function in asthma and to undertake a meta-analysis where similar data were available.

\section{Methods}

\section{Search strategy}

We conducted systematic searches of the PubMed, EMBASE and CENTRAL databases from inception to 22 July 2019 for peer-reviewed studies. For full details refer to table e1. Reference lists of related review articles were manually screened for additional studies meeting our inclusion criteria.

\section{Eligibility criteria}

We included randomised controlled trials (RCTs) and observational studies with $\geqslant 1$ year follow-up in which the effects of maintenance ICS on change in lung function (growth or decline) and risk of fixed airflow obstruction was assessed. Studies involving either children or adults with current asthma were included. Studies were required to have an appropriate placebo or control comparison group. Inclusion was limited to English-language studies published in full text. Studies with concomitant use of maintenance systemic corticosteroids were excluded.

A range of definitions of asthma were accepted, including physician-diagnosed asthma, spirometrically defined asthma and survey-reported asthma. Current asthma was defined as: asthma symptoms or asthma-related healthcare utilisation within the last 12 months.

\section{Outcomes of interest}

There were two outcomes of interest. 1) Change in lung function from baseline defined as: change in forced expiratory volume in $1 \mathrm{~s}\left(\mathrm{FEV}_{1}\right), \mathrm{FEV}_{1} /$ forced vital capacity (FVC), forced expiratory flow at $25-75 \%$ of $\mathrm{FVC}\left(\mathrm{FEF}_{25-75 \%}\right)$ or bronchial hyperresponsiveness (BHR). 2) Fixed airflow obstruction defined as post-bronchodilator (BD) $\mathrm{FEV}_{1} / \mathrm{FVC}$ ratio $<0.7$ or less than the lower limit of normal.

\section{Selection of studies}

All studies identified in the search strategy were independently screened by two review authors (D.J. Tan and either D.S. Bui or X. Dai). Full texts of all studies considered eligible, potentially eligible or unclear

Affiliations: ${ }^{1}$ Allergy and Lung Health Unit, Centre for Epidemiology and Biostatistics, School of Population and Global Health, University of Melbourne, Melbourne, Australia. ${ }^{2}$ Faculty of Medicine, University of New South Wales, Randwick, Australia. ${ }^{3}$ National Health and Lung Institute, Imperial College London, London, UK. ${ }^{4}$ School of Public Health \& Preventive Medicine, Monash University, Melbourne, Australia. ${ }^{5}$ School of

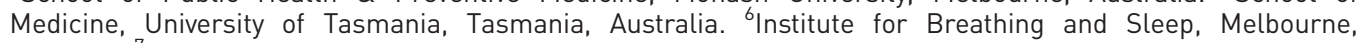
Australia. ${ }^{7}$ Equal senior authors.

Correspondence: Shyamali Dharmage, Allergy and Lung Health Unit, Centre for Epidemiology and Biostatistics, School of Population and Global Health, University of Melbourne, Level 3, 207 Bouverie Street, Parkville 3052, Victoria, Australia. E-mail: s.dharmagedunimelb.edu.au 
were retrieved and assessed. Disagreements at this stage or further on were settled by consultation with a third author (D.S. Bui or X. Dai).

\section{Data extraction}

Using a standardised data extraction form, study characteristics and outcome data were extracted independently by two review authors (D.J. Tan and either D.S. Bui or X. Dai). Information extracted included the first author, date of publication, study design, study setting, date of study, number of participants, mean age, age range, sex, asthma definition, asthma severity, smoking history, ICS use (type, frequency, dose and duration), outcome definitions, confounders and interactions, effect estimates and 95\% confidence intervals.

\section{Quality assessment and risk of bias}

Risk of bias was assessed independently by two review authors (D.J. Tan and either D.S. Bui or X. Dai) using the Cochrane Collaboration's Tool for individual RCTs, modified Newcastle-Ottowa scale (NOS) for individual observational studies [14], and GRADE (Grading of Recommendations Assessment, Development and Evaluation) guidelines for quality by outcome across a range of studies [15].

\section{Statistical analysis}

Where multiple studies reported data for a single outcome on different scales (e.g. \% predicted or $\mathrm{mL}$ ), standardised mean differences (SMDs) were calculated to enable pooling of the data. If studies presented data in both formats, data presented as \% predicted were selected in preference to $\mathrm{mL}$ for conversion to SMD.

RCTs and observational studies were meta-analysed separately. Observational studies were required to have adjusted for asthma severity and smoking status in the statistical analysis or have accounted for these factors in their study design (e.g. inclusion criteria of only mild asthma) to be included in the meta-analysis.

Heterogeneity was assessed using the $\mathrm{I}^{2}$ statistic. Fixed-effects models were used if $\mathrm{I}^{2}$ was $<25 \%$. Random-effects models were used if $\mathrm{I}^{2}$ was between $25 \%$ and $75 \%$. Pooled estimates were presented but considered to be unreliable if $\mathrm{I}^{2}$ was $>75 \%$. The selected model for each main outcome was then applied to any related subgroup meta-analyses. Data were pooled using the program Review Manager, version 5.3 [16].

\section{Subgroup analyses}

The following planned subgroup analyses were performed where possible: 1) age group: children (aged $<18$ years) versus adults (aged $\geqslant 18$ years); 2) atopic status: atopic versus nonatopic; 3) blood or sputum eosinophil level: high versus low; threshold as specified by the individual studies; 4) smoking status: current versus never- or former smoker; 5) length of follow-up: RCTs: 1 year versus $>1$ year; observational studies: 10 years or $<10$ versus $>10$ years.

\section{Sensitivity analyses}

The follow sensitivity analyses were performed where possible: 1) risk of bias assessments: with versus without studies considered at high risk of bias; 2) meta-analysis methodology: fixed-effects models versus random-effects models.

\section{Protocol registration}

The protocol for this systematic review was registered prospectively in the PROSPERO database in September 2017 (registration number: CRD42017053543). FVC and BHR were added as outcomes of interest after protocol registration.

\section{Results}

\section{Search results}

6517 records were identified from the database searches and reference lists of related articles (figure 1). Of these, 365 records were selected for full-text screening and of these, 38 articles from 24 unique studies met the pre-specified inclusion criteria. Excluded records and their reasons for exclusion are listed in table e2. Characteristics of the included studies have been summarised in table 1 (RCTs) and table e3 (observational studies).

\section{RCTs}

Included studies

13 RCTs with a total of 11678 participants were included [12, 17-27]. Five RCTs were performed in children $(\mathrm{n}=1250)[18,21,23,26,27]$, seven in adults $(\mathrm{n}=3263)[12,17,19,20,22,24,25]$ and one in both children and adults $(n=7165)$ [3]. Three studies contributed $90 \%$ of the participants (START: $n=7165$; SYGMA1: 


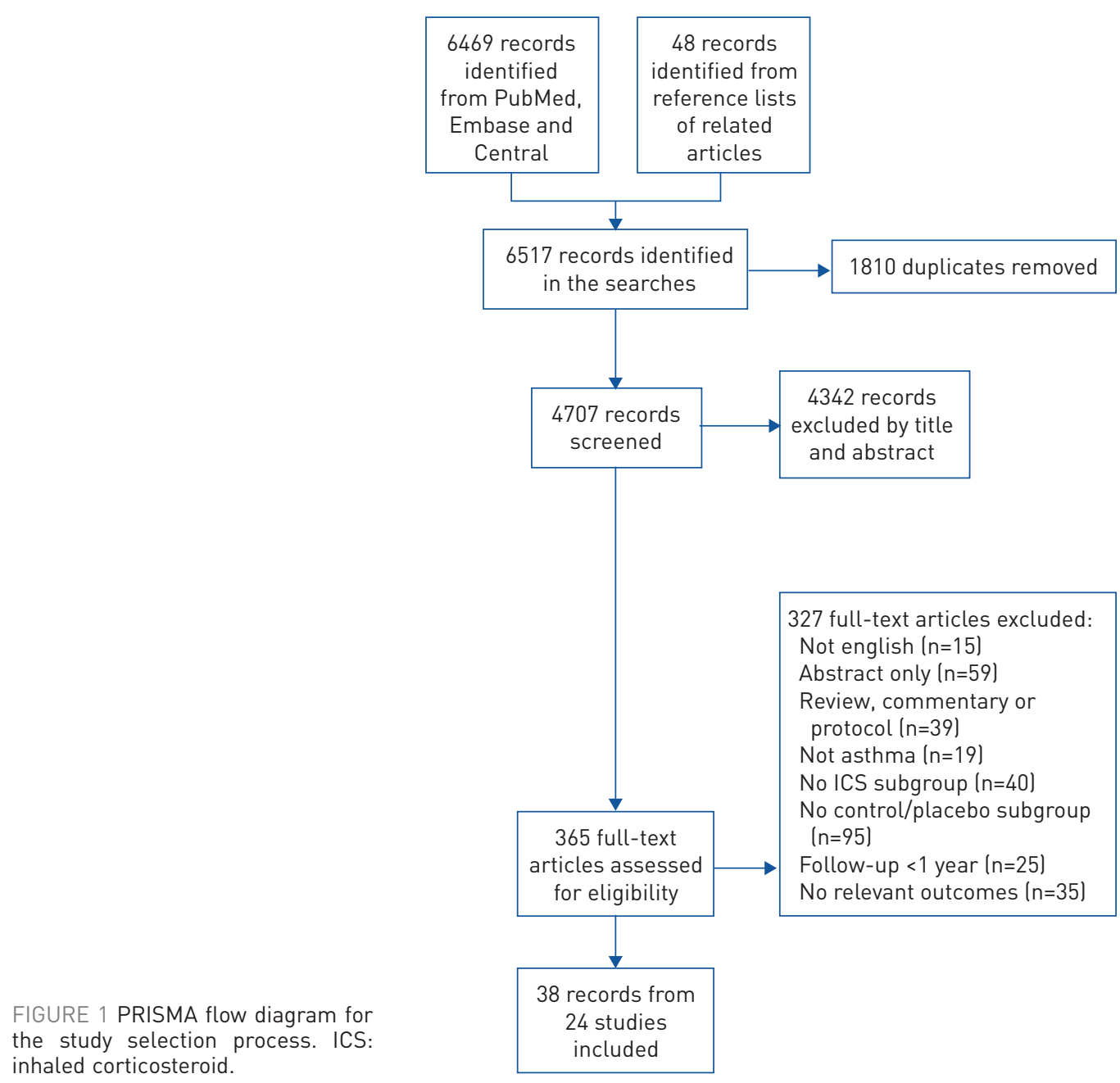

$\mathrm{n}=2559$; CAMP: $\mathrm{n}=729$ ) $[3,24,27]$. Asthma was physician-diagnosed in all studies. BHR or bronchodilator reversibility was an additional inclusion criterion in nine studies $(n=10889)$ [3, 19, 20, 22-27]. Seven studies were in individuals with mild asthma $(\mathrm{n}=10708)[3,17-19,21,24,26]$, two were in individuals with mild-to-moderate asthma $(\mathrm{n}=764)[12,27]$, one was in individuals with moderate-to-severe asthma $(\mathrm{n}=54)$ [23], and three did not limit inclusion by asthma severity $(n=152)[20,22,25]$. Eight studies used budesonide $(\mathrm{n}=11128)[3,17,21-25,27]$, three used fluticasone $(\mathrm{n}=149)[12,19,20]$ and two used beclomethasone $(\mathrm{n}=401)[18,26]$. Based on GINA criteria [11], ICS were categorised as low dose in eight studies $(\mathrm{n}=10459)$ $[3,17,19-22,24,25]$, medium dose in four studies $(\mathrm{n}=1184)[18,23,26,27]$ and high dose in one study $(\mathrm{n}=35)$ [12]. Median (interquartile range) follow-up was $1.0(1-4)$ years.

Risk of bias assessments

Methodological quality in the RCTs was good overall (figure 2). All RCTs were assessed to be at low risk of selection, performance and detection bias. Five studies were assessed to be at high risk of attrition bias, related to high dropout rates [19-21, 23, 25]. Reporting bias was unclear in eight mainly older studies for which prospective protocols were not available [18-23, 25, 26]. GRADE certainty of evidence across outcomes ranged from low to high as outlined in tables 2 and 3.

\section{Studies excluded from the quantitative analysis}

Two small RCTs were excluded from the quantitative analysis due to methodological factors or nonrepresentative study samples $[20,23]$. One study compared budesonide to placebo in 47 children with moderate-to-severe asthma, but had significant and selective dropout in the placebo arm, which resulted in early discontinuation [23]. The budesonide arm was continued to study completion at 3 years and the authors did not account for differences in treatment duration in the comparisons. The second study was published as a brief report and compared fluticasone to placebo in 45 adults with asthma and accelerated 
TABLE 1 Study characteristics of the included randomised controlled trials

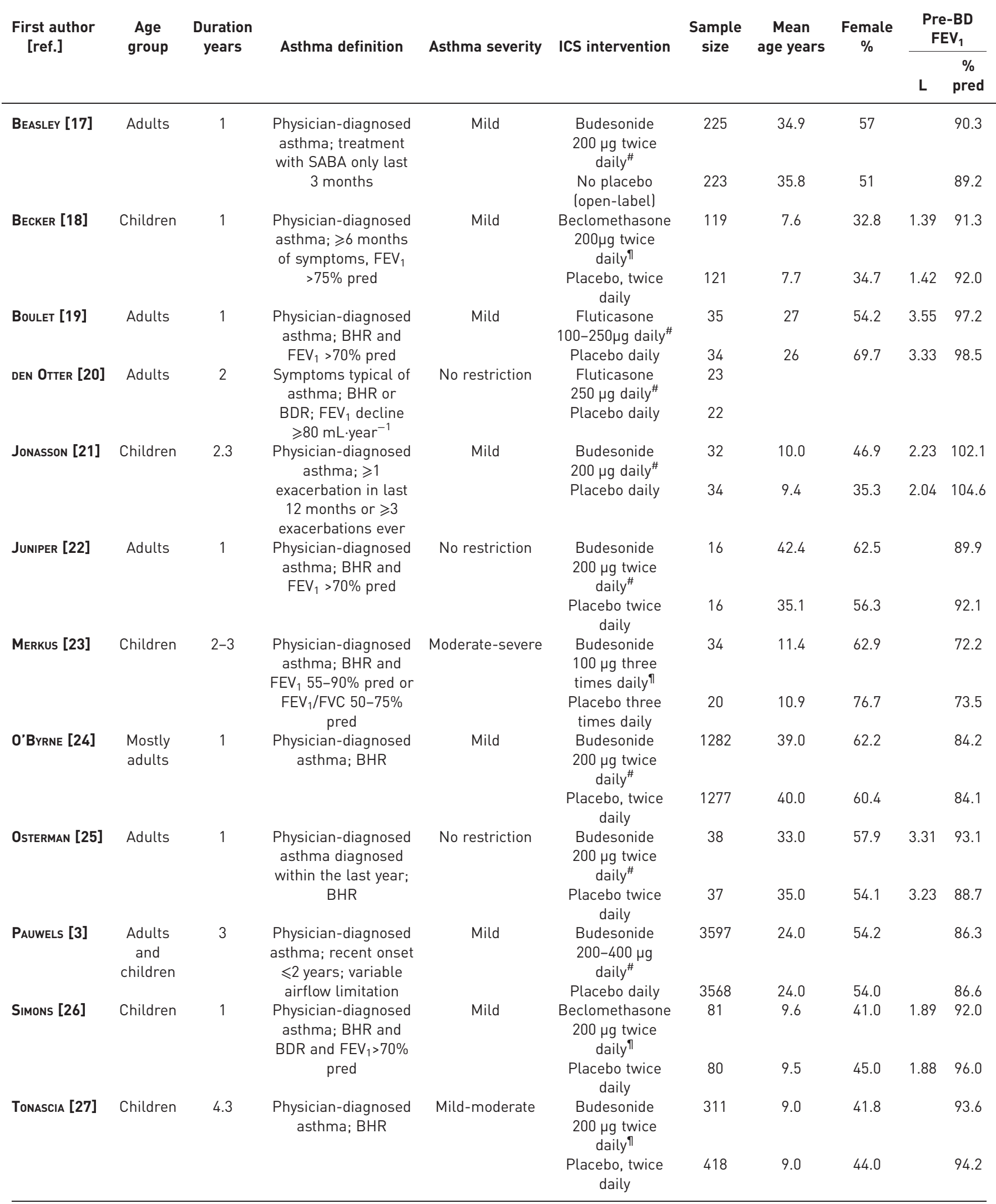




\begin{tabular}{|c|c|c|c|c|c|c|c|c|c|c|}
\hline $\begin{array}{l}\text { First author } \\
\text { [ref.] }\end{array}$ & $\begin{array}{l}\text { Age } \\
\text { group }\end{array}$ & $\begin{array}{l}\text { Duration } \\
\text { years }\end{array}$ & Asthma definition & Asthma severity & ICS intervention & $\begin{array}{l}\text { Sample } \\
\text { size }\end{array}$ & $\begin{array}{c}\text { Mean } \\
\text { age years }\end{array}$ & $\begin{array}{c}\text { Female } \\
\%\end{array}$ & \multicolumn{2}{|c|}{$\begin{array}{l}\text { Pre-BD } \\
\text { FEV }_{1}\end{array}$} \\
\hline \multirow[t]{2}{*}{$W_{\text {ARD }}[12]$} & Adults & 1 & $\begin{array}{l}\text { Physician-diagnosed } \\
\text { asthma; positive skin } \\
\text { prick testing to } \geqslant 3\end{array}$ & Mild-moderate & $\begin{array}{c}\text { Fluticasone } \\
750 \mu g \text { twice } \\
\text { daily }^{+}\end{array}$ & 17 & & & & 96.0 \\
\hline & & & $\begin{array}{c}\text { common } \\
\text { aeroallergens }\end{array}$ & & $\begin{array}{c}\text { Placebo twice } \\
\text { daily }\end{array}$ & 18 & & & & 94.0 \\
\hline
\end{tabular}

ICS: inhaled corticosteroid; BD: bronchodilator; $\mathrm{FEV}_{1}$ : forced expiratory volume in $1 \mathrm{~s}$; SABA: short-acting $\beta_{2}$-agonist; BHR: bronchial

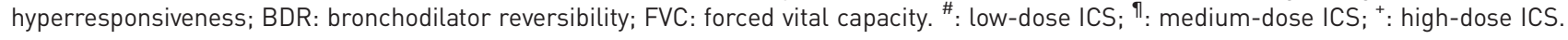

lung function decline $\left(>80 \mathrm{~mL} \cdot \mathrm{year}^{-1}\right)$ [20]. The study sample was considered to be highly selected due the declining lung function inclusion criteria and not representative of the general asthma population.

\section{Pre-BD lung function}

Pre-BD FEV

Eight studies measured changes in pre-BD $\mathrm{FEV}_{1}$ (\% pred) (table 2, figure 3) [3, 12, 19, 21, 22, 25-27]. Improvements were observed with treatment across all age groups (2.22\% (95\% CI 1.32-3.12), $\mathrm{n}=8332$ ), with similar estimates of strength in association in children $(2.08 \%(95 \%$ CI $0.71-3.44), \mathrm{n}=4151)$ and adults $(2.47 \%$ (95\% CI 1.64-3.29), n=4181). Findings were similar in four studies which measured pre-BD $\mathrm{FEV}_{1}$ in $\mathrm{mL}(74 \mathrm{~mL}(95 \% \mathrm{CI} 54-94), \mathrm{n}=3603)[18,24,25,27]$ and when SMDs were calculated and pooled (0.21 SMD (95\% CI 0.12-0.30), $\mathrm{n}=11$ 131).

\section{Pre-BD FVC}

Two studies measured changes in pre-BD FVC (\% pred) and found no evidence of a treatment benefit $(0.10 \%$ (95\% CI $-1.23-1.43), \mathrm{n}=804)[25,27]$. Findings were similar in two studies which reported pre-BD FVC in $\mathrm{mL}(-13 \mathrm{~mL}(95 \% \mathrm{CI}-149-124), \mathrm{n}=804)[25,27]$ and when SMD were calculated and pooled (0.01 SMD (95\% CI -0.13-0.15), $\mathrm{n}=804)$

\section{Pre-BD FEV $/$ /FVC}

No studies measured changes in pre-BD $\mathrm{FEV}_{1} / \mathrm{FVC}$ (\% pred). One study performed in children measured change in pre-BD $\mathrm{FEV}_{1} / \mathrm{FVC}$ (ratio multiplied by 100) and found significant improvements with treatment (1.60 (95\% CI 0.64-2.56), $\mathrm{n}=729)$ [27].

\section{Pre-BD FEF $25-75$}

One study measured changes in pre- $\mathrm{BD} \mathrm{FEF}_{25-75 \%}$, but was excluded from the quantitative analysis due to concerns around methodology [23]. The study reported benefits in pre-BD $\mathrm{FEV}_{25-75 \%}$ with ICS use over the treatment period.

\section{Post-BD lung function \\ Post-BD FEV,}

One study in adults [3] and two in children $[3,27]$ measured changes in post-BD FEV $1 \%$ pred) (table 3, figure 4). Significant improvements were observed with treatment in adults (1.54\% (95\% CI 0.87-2.21), $\mathrm{n}=3970$ ), but not in children $(0.20 \%$ (95\% CI -0.49-0.90), $\mathrm{n}=3924)$ ( $\mathrm{p}$-subgroup differences0.006). One study reported change in post-BD $\mathrm{FEV}_{1}(\mathrm{~mL})$ in children and also did not demonstrate a treatment benefit ( $-40 \mathrm{~mL}$ (95\% CI $-115-35, \mathrm{n}=729)$ [27]. Findings were similar when SMD were calculated and pooled, with benefits found in adults (0.14 SMD (95\% CI 0.08-0.21), $\mathrm{n}=3970)$, but not in children (0.02 SMD (95\% CI -0.05-0.09), $\mathrm{n}=4924)$.

\section{Post-BD FVC}

One study performed in children measured changes in post-BD FVC (\% pred) and found no evidence of treatment benefit $(-0.20 \%(95 \% \mathrm{CI}-1.40-1.00), \mathrm{n}=729)$ [27]. The same study also measured changes in post-BD FVC $(\mathrm{mL})$ and a borderline adverse effect of treatment would have been reported if this measure was used $(-60 \mathrm{~mL}(95 \% \mathrm{CI}-120--0), \mathrm{n}=729)$ [27]. 


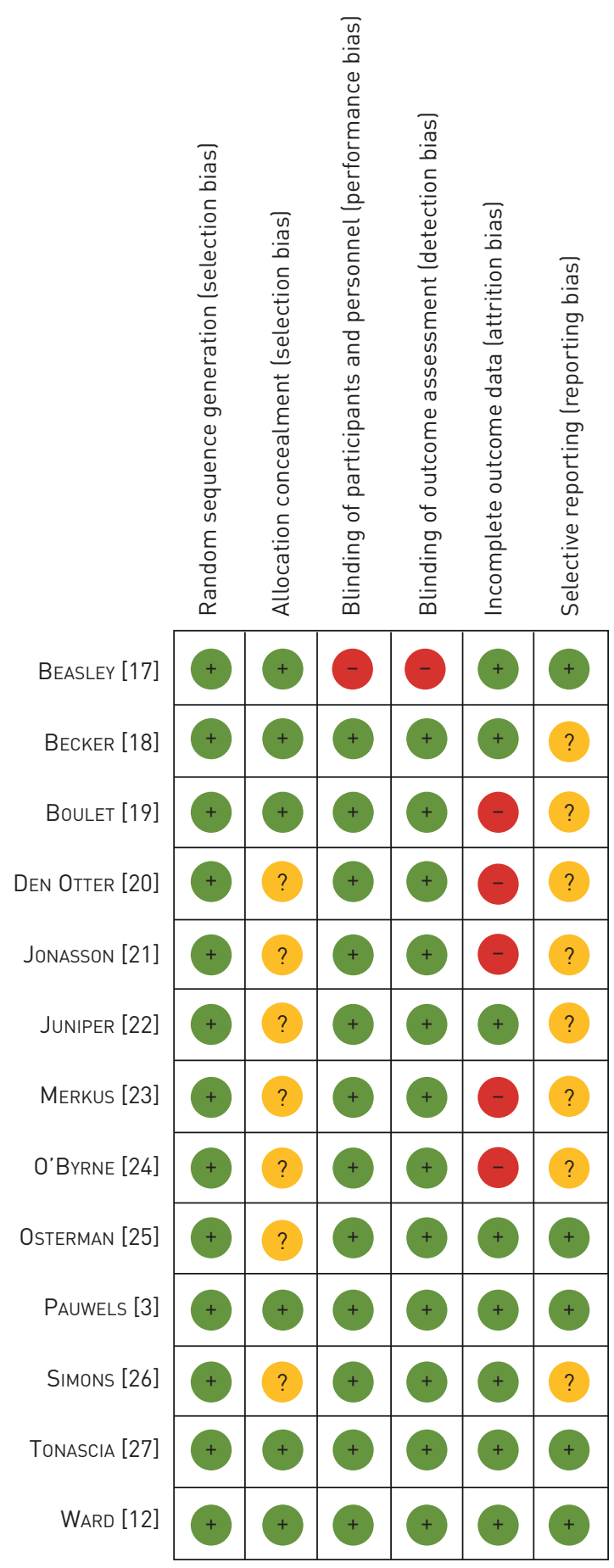

FIGURE 2 Cochrane Collaboration Tool (randomised controlled trials): risk of bias assessments for each included study.

\section{Post-BD FEV $/$ /FVC}

No studies measured changes in post-BD $\mathrm{FEV}_{1} / \mathrm{FVC}$ (\% pred). One study in children measured change in post-BD FEV1/FVC (ratio) and found an association in the direction of a benefit $(0.70$ (95\% CI -0.081.48), $\mathrm{n}=729$ ), although this did not reach statistical significance [27].

Post-BD FEF $25-75 \%$

One study measured changes in post-BD $\mathrm{FEF}_{25-75 \%}$, but was excluded from the quantitative analysis due to methodological factors [23]. The study reported benefits in post-BD $\mathrm{FEF}_{25-75 \%}$ with ICS use over the treatment period. 
TABLE 2 Meta-analysis of randomised controlled trials for pre-bronchodilator (BD) outcomes stratified by age group

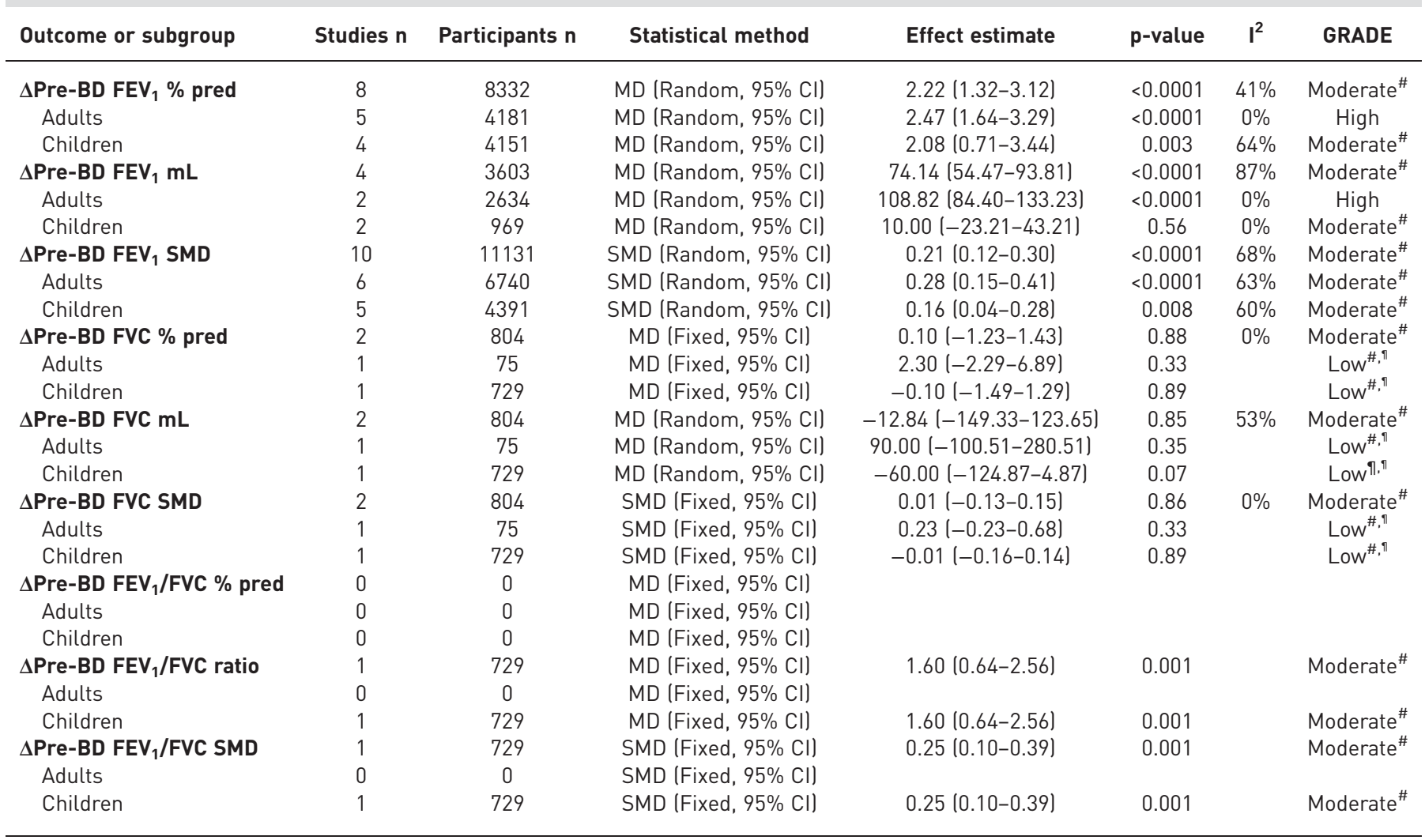

$\mathrm{FEV}_{1}$ : forced expiratory volume in $1 \mathrm{~s}$; FVC: forced vital capacity; MD: mean difference; SMD: standardised mean difference. ${ }^{\#}$ : GRADE score downgraded for heterogeneity or inconsistency of results between studies; ": GRADE score downgraded for imprecision, $95 \% \mathrm{Cl}$ includes important benefit and potential harm.

\section{Fixed airflow obstruction}

There were no RCTs that reported data on incidence of fixed airflow obstruction.

\section{BHR}

Three studies measured $\mathrm{BHR}$ as change in doubling concentrations of methacholine provocative concentration causing a $20 \%$ fall in $\mathrm{FEV}_{1}\left(\mathrm{PC}_{20}\right)[19,21,22]$. Treatment increased methacholine $\mathrm{PC}_{20}$ compared to placebo (1.07 doubling concentrations (95\% CI 0.65-1.49), $\mathrm{n}=223$ ). Findings were similar when BHR was expressed as change in methacholine $\mathrm{PC}_{20} \mathrm{mg} \cdot \mathrm{mL}^{-1}(0.99$ (95\% CI 0.32-1.66), $\mathrm{n}=161)$ [26], change in methacholine $\mathrm{PC}_{20}$ factor increase from baseline (1.10× (95\% CI 0.61-1.59), $\left.\mathrm{n}=729\right)$ [27] and change in histamine $\mathrm{PC}_{20}$ factor increase from baseline (2.59× (95\% CI 1.18-4.00), $\left.\mathrm{n}=75\right)$ [25].

\section{Subgroup analyses}

Stratification by smoking status

The largest study (START) reported changes in pre-BD and post-BD lung function stratified by smoking status. No significant differences were found between smokers and nonsmokers for change in either pre-BD $\mathrm{FEV}_{1}$ (p-subgroup difference0.22) or post-BD FEV (p-subgroup difference0.37) [3].

Stratification by duration of follow-up

When stratified by duration of follow-up, the greatest benefits for pre-BD $\mathrm{FEV}_{1}$ were found in the first year of follow-up $[18,19,22,24,25]$ when compared to studies with follow-up $>1$ year $[3,27]$ (p-subgroup differences0.004) (figure e1). Stratification for post-BD lung function by follow-up could not be performed as all studies had follow-up durations $>1$ year.

Other pre-specified subgroup analyses

No studies provided outcome data for the other pre-specified subgroup analyses by atopic status, blood or sputum eosinophil counts. 
TABLE 3 Meta-analysis of randomised controlled trials for post-bronchodilator (BD) outcomes stratified by age group

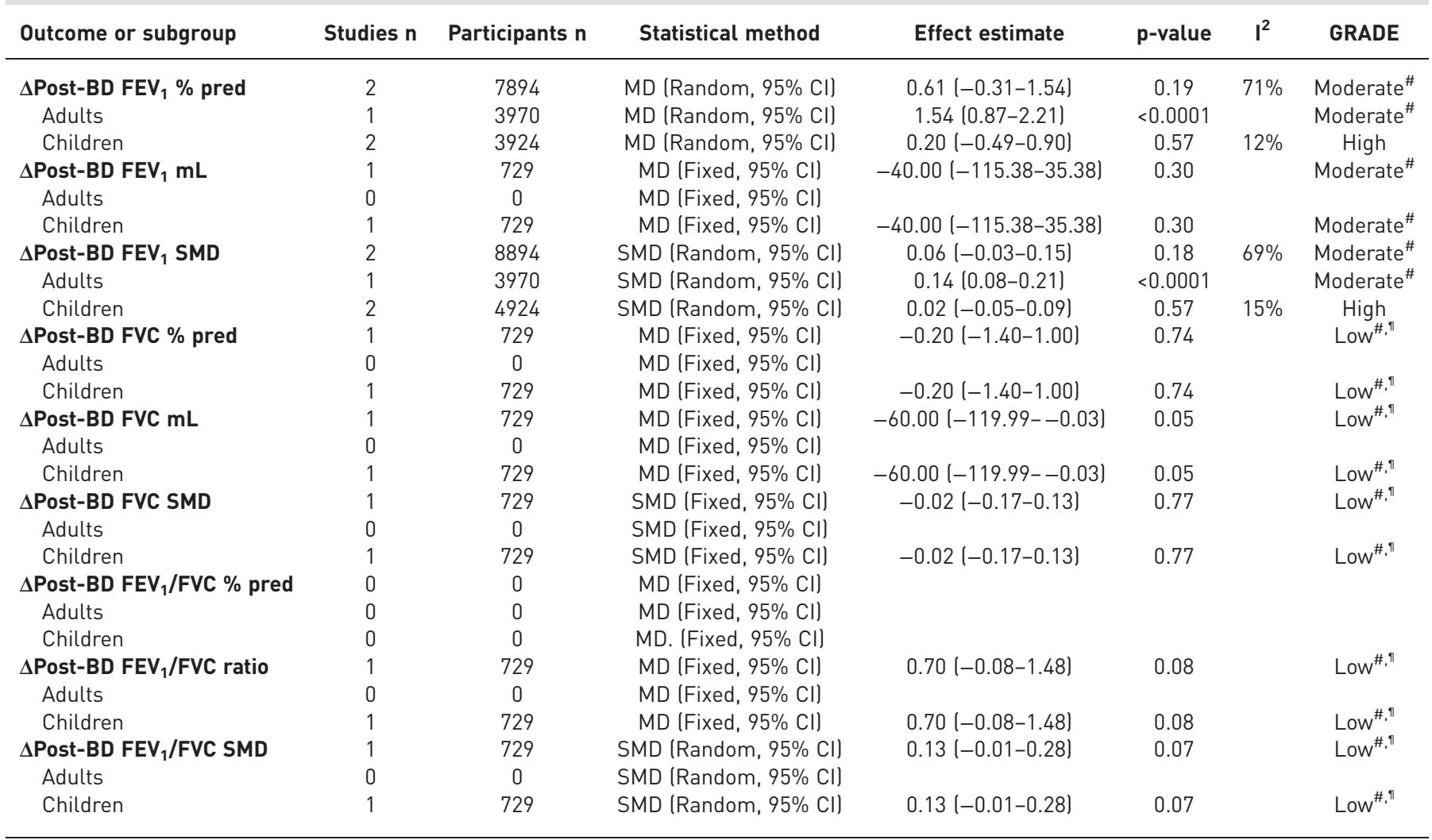

$\mathrm{FEV}_{1}$ : forced expiratory volume in $1 \mathrm{~s} ; \mathrm{FVC}$ : forced vital capacity; MD: mean difference; SMD: standardised mean difference. ${ }^{\#}$ : GRADE score downgraded for heterogeneity or inconsistency of results between studies; ": GRADE score downgraded for imprecision, $95 \% \mathrm{Cl}$ includes important benefit and potential harm.

Sensitivity analyses

Sensitivity analyses performed by meta-analysis model and risk of bias assessment did not significantly affect the results for pre-BD or post-BD lung function (table e4).

\section{Observational studies \\ Included studies}

11 observational studies with a total of 3720 participants were included [28-37]. Four studies were in children $(n=787)[28,30,34,36]$ and seven in adults $(n=2933)[29,31-33,35,37,38]$. Asthma was physician-diagnosed in eight studies $(\mathrm{n}=1044)$ [28, 30-34, 36, 37] and self-reported in three studies $(n=2676)[29,35,38]$. ICS use was measured at baseline in five studies $(n=750)[28,31,32,34,36]$, at follow-up in four studies $(\mathrm{n}=2663)[29,30,37,38]$, and at both baseline and follow-up in two studies $(\mathrm{n}=307)[33,35]$. BHR or bronchodilator reversibility was required as an additional inclusion criterion in five studies $(\mathrm{n}=2108)[29,31,32,34,37]$. Asthma was of mild-to-moderate severity in two studies $(\mathrm{n}=301)$ $[28,37]$ and the remaining nine did not restrict inclusion by asthma severity $(\mathrm{n}=3419)$. Median (interquartile range) follow-up was 8.4 (3-28) years.

\section{Quality assessment}

Methodological quality of the cohort studies varied (table e5), with a median (interquartile range) NOS score of 7 (4-9). Three studies were assessed as being of "very good" quality (NOS $\geqslant 9)[35,36,38]$, three of "good" quality (NOS 7-8) [28, 31,33], four of "satisfactory" quality (NOS 5-6) [29, 32, 34, 37], and one of "unsatisfactory" quality (NOS 0-4) [30]. Quality was most often negatively impacted by inadequate adjustment for critical confounders (i.e. asthma severity and smoking status). Six studies also had high rates of loss to follow-up or selective patterns of dropout in the comparison groups $[29,30,33,35,37,38]$. 


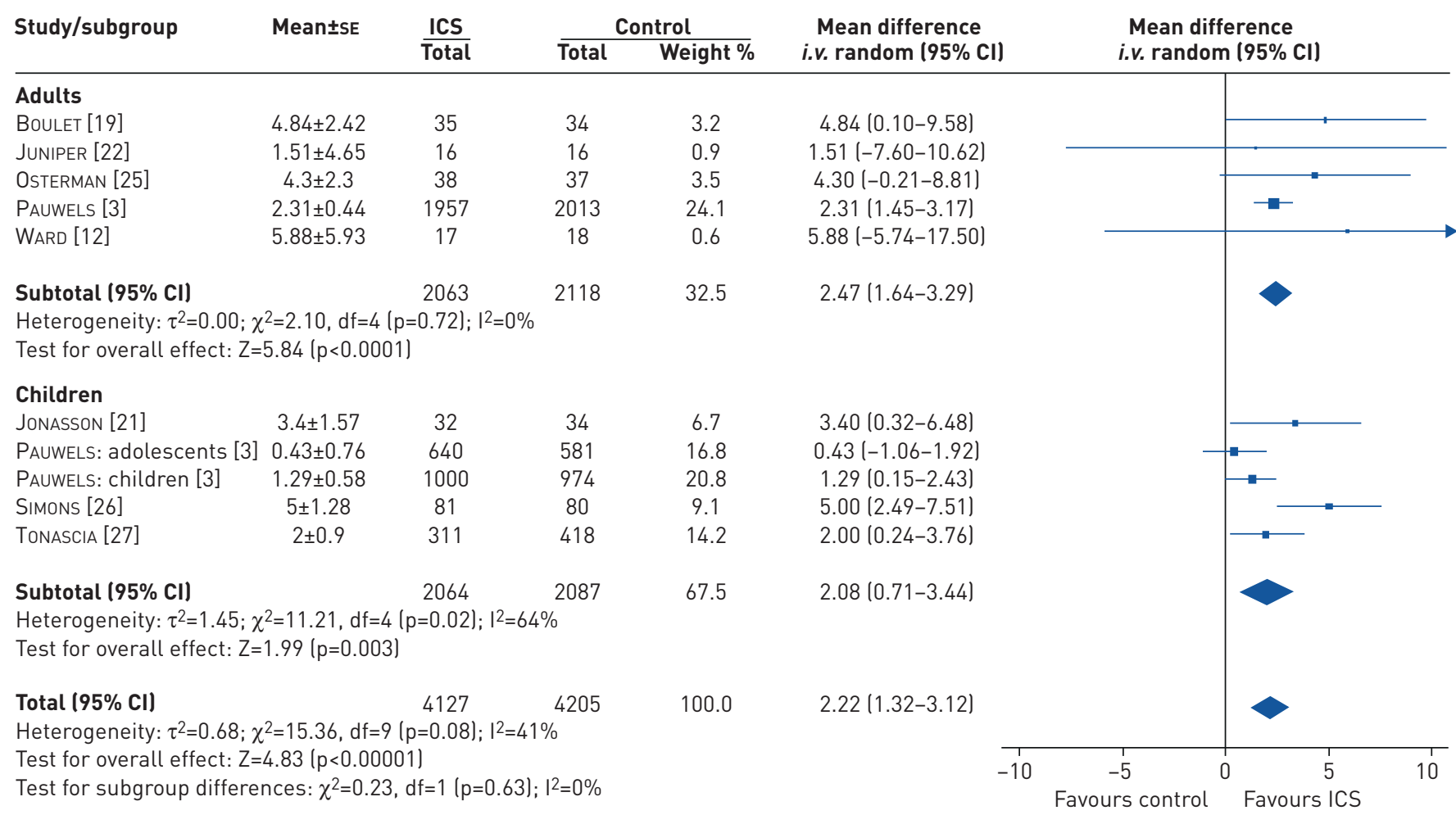

FIGURE 3 Forest plot comparison (randomised controlled trials): change in pre-bronchodilator forced expiratory volume in $1 \mathrm{~s}$ (\% predicted) stratified by age. ICS: inhaled corticosteroid.

\section{Studies excluded from the quantitative analysis}

Seven observational studies were excluded from the quantitative analysis as critical confounders (asthma severity and smoking status) were not adjusted for in the statistical analysis or through study design [28-32, 34, 37]. Sensitivity analyses with these studies included are provided in table e6. Of the remaining four studies, NOS assessments ranged from 8 to $10[33,35,36,38]$.

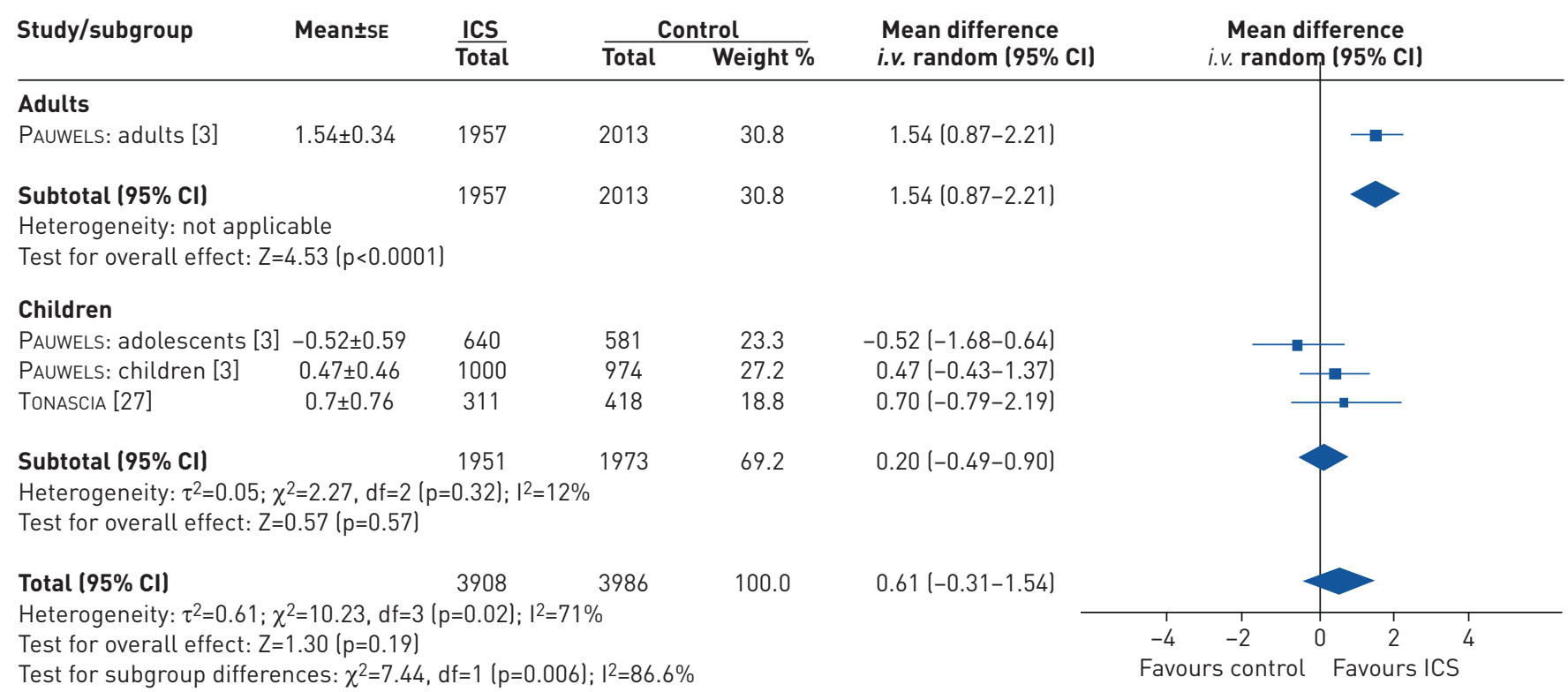

FIGURE 4 Forest plot comparison (randomised controlled trials): change in post-bronchodilator forced expiratory volume in $1 \mathrm{~s}$ (\% predicted) stratified by age. ICS: inhaled corticosteroid. 


\section{Pre-BD lung function}

Sufficient data to perform a meta-analysis were available for only one outcome, pre-BD $\mathrm{FEV}_{1}(\mathrm{~mL})$, with three studies, all performed in adults, finding significant per-year treatment benefits over their follow-up periods $\left(14 \mathrm{~mL} \cdot \mathrm{year}^{-1}\right.$ (95\% CI 2-26), $\left.\mathrm{n}=939\right)$ [33, 35, 38]. One study, performed in children also found treatment benefits in pre-BD $\mathrm{FEV}_{1}$ (\% pred) $(0.81 \%$ per year $(95 \%$ CI $0.01-1.61), \mathrm{n}=190)$ [36].

\section{Post-BD lung function}

None of the four studies measured post-BD outcomes or incidence of fixed airflow obstruction.

\section{Subgroup analyses}

Stratification by smoking status

One observational study reported data on pre-BD $\mathrm{FEV}_{1}$ stratified by smoking status [35]. Similar to the START trial, this study found no significant differences between smokers and nonsmokers.

\section{Other pre-specific subgroup analyses}

No observational studies provided data for other pre-specified subgroup analyses by atopic status, blood or sputum eosinophil counts, or duration of follow-up.

\section{Sensitivity analyses}

Sensitivity analyses performed by meta-analysis model and risk of bias assessment did not significantly affect the results for pre-BD outcomes (table e6). There were insufficient studies to perform sensitivity analyses for post-BD outcomes.

\section{Discussion}

This is the first systematic review to comprehensively evaluate the long-term effects of ICS on lung function in patients with asthma. Within the RCTs, maintenance ICS was associated with modest improvements in pre-BD $\mathrm{FEV}_{1}$ across all age groups, whereas improvements in post-BD $\mathrm{FEV}_{1}$ were observed only in adults. The greatest benefits were observed in the first year of treatment, and estimates were similar between smokers and nonsmokers. Due to the characteristics of the included studies, these findings are most applicable to children and adults with mild asthma treated with low-dose ICS. However, we found no RCT data on the effect of ICS on incidence of fixed airflow obstruction in asthma. Moreover, while the observational studies were generally of longer duration, many were assessed as being at high risk of bias due to inadequate adjustment for confounding factors.

Current asthma guidelines recommend ICS as a first-line option for persistent asthma. ICS therapy improves asthma control, reduces exacerbations and provides a modest increase in short-term measures of lung function over weeks to months $[2,3,39,40]$. While we found these benefits on lung function persisted beyond the first year of treatment, the magnitude of benefit was relatively modest and below conventional minimally clinically important difference cut-offs for $\mathrm{FEV}_{1}(230 \mathrm{~mL})$ [41]. Additionally, in the subgroup analysis by duration of follow-up, the greatest benefits were obtained within the first year and then appeared to decrease with time. This trend was similarly observed in START (year 1 versus year 3) and CAMP (year 1 versus year 4) [3, 27]. However, it should be noted that this observation was confounded by several time-dependent factors, including decreasing compliance to ICS with time in the intervention arms and increasing use of nonintervention asthma medications in the placebo arms [3, 24, 27]. Therefore, rather than a cumulative benefit on lung function over time in these mild asthma trials, we found that maintenance ICS use was associated with a modest absolute benefit which was maintained to an extent with continued treatment. These effects could be more pronounced in patients with moderate-to-severe disease and in those receiving higher-dose ICS. However, there were limited data to evaluate such effects in these subgroups.

The age-based differences for post-BD $\mathrm{FEV}_{1}$ were unexpected. In meta-analyses with small numbers of contributing trials, subgroup differences can arise from subtle differences in methodology and this was important to exclude. However, the majority of the data for this outcome, for both children and adults, came from one large study (START), which recruited patients using standardised methodology across age groups [3]. CAMP also contributed data on post-BD $\mathrm{FEV}_{1}$ and did not identify a treatment benefit in children [27], despite their participants having a slightly higher disease severity at baseline than participants in START (mild-to-moderate versus mild only) [27]. While there appeared to be a trend toward a benefit with treatment on post-BD $\mathrm{FEV}_{1} / \mathrm{FVC}$ in CAMP, this did not reach statistical significance.

Several reasons may account for these age-based differences. First, the diagnosis of asthma in children is often less clear than in adults and physician-diagnosed asthma in the absence of objective tests can be uncertain $[42,43]$. Inclusion of children who may not have benefited from ICS in the first place, such as those with viral-induced or episodic wheeze, would have reduced any observed treatment benefit. 
Secondly, childhood asthma often improves over adolescence and treatment differences could also have been reduced by improved asthma control or asthma remission as part of the natural course [44]. This theory is supported by data from START, in which adolescents (aged 11-17 years) were less likely to benefit from maintenance ICS compared to both younger children (aged 5-10 years) and adults (aged 1866 years). Lung function trajectory studies have also shown that small deficits established early in life can diminish with time, with some children with below-normal lung function entering a "catch-up" phase during puberty $[7,45]$. Consequently, rapid growth in lung function during childhood might also explain the observed treatment differences between children and adults.

While the observational studies had longer durations of follow-up compared to the RCTs, few met pre-specified criteria to be included in the quantitative analysis. Of those included, ICS use was variably assessed at baseline and/or follow-up, and change in medication use between these time-points were not clearly assessed. Acknowledging these important limitations, ICS use as defined in these observational studies was associated with improved trends in pre-BD $\mathrm{FEV}_{1}$ across the follow-up periods, which ranged from 9 to 13 years. The observational studies were also more likely to have observed any effects of ICSs on fixed airflow obstruction in asthma, which may develop over years to decades [7]. Unfortunately, we did not identify any studies reporting data for this outcome.

The effects of ICSs on lung function in asthma appear to be mediated, at least in part, through an effect on asthma exacerbations. Exacerbations represent intermittent periods of intense airways inflammation, and have been associated with structural airways remodelling and rapid lung function decline [46, 47]. In a post hoc analysis of START, O'BYRNE et al. [47] showed that low-dose budesonide attenuated these adverse effects, with treatment associated with a reduction in both the rate and impact of severe exacerbations on post-BD $\mathrm{FEV}_{1}$. While budesonide was not found to improve post-BD $\mathrm{FEV}_{1}$ in children in the overall analysis, treatment was associated with a significant reduction in post-BD $\mathrm{FEV}_{1}$ impairment in children who experienced one or more severe exacerbations during the 3 -year follow-up $(-2.38 \%$ predicted on budesonide versus $-6.29 \%$ predicted on placebo). This interaction, also observed in adults, supports the hypothesis that treatment effects are likely to be greater in patients with more severe disease.

Following completion of the recent SYGMA1 and Novel START trials, there is now strong evidence on the benefits of maintenance ICSs compared to short-acting $\beta$-agonist (SABA) reliever monotherapy for exacerbation prevention in mild asthma [17, 24]. Accordingly, the 2019 GINA update has now recommended against SABA reliever monotherapy in mild asthma, and instead recommends ICS containing treatment for all mild asthma patients. With new evidence supporting the use of as-needed budesonide-formoterol as an alternative to maintenance ICS in step 2 treatment $[13,17,24,48]$, the relative effects of these regimens on long-term lung function should also be examined.

This systematic review has a number of key strengths. We performed comprehensive searches of three electronic databases and two authors independently screened references, extracted data and performed quality assessments. We also evaluated both experimental and observational evidence. The RCTs provided higher quality evidence but recruited highly selected samples of asthma patients suited for drug efficacy trials. In contrast, the observational studies evaluated a broader range of asthma patients over longer durations of follow-up. Given the strong existing evidence base and ethical implications, we believe there are unlikely to be further placebo-controlled trials conducted. However, high-quality observational data on the longer term effects of ICS treatment in moderate-to-severe asthma, particularly for post-BD lung function, are still required

This review also had several limitations. First, one of our objectives of examining the effect of ICS on incidence of fixed airflow obstruction in asthma could not be achieved due to the lack of available evidence. Secondly, our review was limited to English language publications and relevant non-English studies may have been excluded. Thirdly, the Cochrane Collaboration now recommends the ROBINS-I tool for assessing risk of bias in nonrandomised studies [49] and while limited observational data were included, risk of bias assessments for these studies were performed using the formerly recommended NOS tool [14]. Fourthly, many of the included studies compared low-dose budesonide to placebo and recruited only patients with mild asthma. Therefore, our results may not be representative of more severe asthma, higher-dose or other ICS agents. Fifthly, most data for several outcomes came from one large RCT (START), which included patients with recent-onset asthma. It is unclear whether outcomes in this sample may have been more pronounced compared to patients with longer term established disease. Finally, most studies focused on changes in $\mathrm{FEV}_{1}$. Other routinely collected spirometry indices (e.g. FVC and $\mathrm{FEV}_{1} /$ FVC) were infrequently reported, which raised concerns of selective reporting for these outcomes.

In conclusion, maintenance ICS are associated with modest, age-dependent improvements in long-term lung function in patients with mild asthma. These improvements represent an added functional benefit to the more accepted clinical actions of ICS and reinforce the importance of ICS as a recommended initial 
treatment option even for mild asthma. Further research is still needed to determine whether ICS use modifies the risk of developing fixed airflow obstruction in asthma, especially in smokers and high-quality observational data from existing prospective cohorts could be used to address these important knowledge gaps. Treatment adherence, correct inhaler technique and correct diagnosis of asthma are factors likely to influence these outcomes in real-world settings.

Author contributions: D.J. Tan, S.C. Dharmage, J.L. Perret, C.J. Lodge, A.J. Lowe, P.S. Thomas, D. Jarvis, M.J. Abramson and E.H. Walters contributed to the study concept and drafted the review protocol. D.J. Tan, D.S. Bui and X. Dai performed the screening, quality assessment and data extraction.

Conflict of interest: D.J. Tan has nothing to disclose. D.S. Bui has nothing to disclose. X. Dai has nothing to disclose. C.J. Lodge has nothing to disclose. A.J. Lowe reports grants from National Health and Medical Research Council, during the conduct of the study. P.S. Thomas reports having participated in advisory board meetings for GSK and Astra Zeneca, unrelated to this work. D. Jarvis has nothing to disclose. M.J. Abramson reports grants from Pfizer and Boehringer Ingelheim, personal fees and non-financial support from Sanofi, and personal fees from GSK, outside the submitted work. E.H. Walters has nothing to disclose. J.L. Perret reports a travel grant from Boehringer-Ingelheim, outside the submitted work. S.C. Dharmage has nothing to disclose.

Support statement: This study was supported by funds from the National Health and Medical Research Council (NHMRC) of Australia European collaborative grant scheme (1101313) as part of ALEC (Ageing Lungs in European Cohorts funded by the European Union's Horizon 2020 Research and Innovation Programme under grant agreement 633212). The funding bodies were not involved in the included work nor the decision to publish. DJT was supported by a NHMRC Postgraduate Scholarship and Royal Australian College of Physicians (RACP) Woolcock Scholarship. Funding information for this article has been deposited with the Crossref Funder Registry.

\section{References}

1 Global Initiative for Asthma. Global Strategy for Asthma Management and Prevention. 2017. https://ginasthma. org/gina-reports/

2 Adams N, Bestall J, Jones PW. Budesonide for chronic asthma in children and adults. Cochrane Database Syst Rev 2001; 4: Cd003274.

3 Pauwels RA, Pedersen S, Busse WW, et al. Early intervention with budesonide in mild persistent asthma: a randomised, double-blind trial. Lancet 2003; 361: 1071-1076.

4 Suissa S, Ernst P, Benayoun S, et al. Low-dose inhaled corticosteroids and the prevention of death from asthma. N Engl J Med 2000; 343: 332-336.

5 Tan DJ, Walters EH, Perret JL, et al. Clinical and functional differences between early-onset and late-onset adult asthma: a population-based Tasmanian Longitudinal Health Study. Thorax 2016; 71: 981-987.

6 Lange P, Celli B, Agusti A, et al. Lung-function trajectories leading to chronic obstructive pulmonary disease. N Engl J Med 2015; 373: 111-122.

7 Bui DS, Lodge CJ, Burgess JA, et al. Childhood predictors of lung function trajectories and future COPD risk: a prospective cohort study from the first to the sixth decade of life. Lancet Respir Med 2018; 6: 535-544.

8 Lange P, Parner J, Vestbo J, et al. A 15-year follow-up study of ventilatory function in adults with asthma. $N$ Engl J Med 1998; 339: 1194-1200.

9 Strunk RC, Weiss ST, Yates KP, et al. Mild to moderate asthma affects lung growth in children and adolescents. J Allergy Clin Immunol 2006; 118: 1040-1047.

10 Agusti A, Faner R, Donaldson G, et al. Chronic Airway Diseases Early Stratification (CADSET): a new ERS Clinical Research Collaboration. Eur Respir J 2019; 53: 1900217.

11 Global Initiative for Asthma. Global Strategy for Asthma Management and Prevention. 2019. https://ginasthma. org/gina-reports/

12 Ward C, Pais M, Bish R, et al. Airway inflammation, basement membrane thickening and bronchial hyperresponsiveness in asthma. Thorax 2002; 57: 309-316.

13 Reddel HK, FitzGerald JM, Bateman ED, et al. GINA 2019: a fundamental change in asthma management: treatment of asthma with short-acting bronchodilators alone is no longer recommended for adults and adolescents. Eur Respir J 2019; 53: 1901046

14 Wells GA, Shea B, O'Connell D, et al. The Newcastle-Ottawa Scale (NOS) for assessing the quality of nonrandomised studies in meta-analyses. PLoS Negl Trop Dis 2013; 7: e2195.

15 Guyatt G, Oxman AD, Akl EA, et al. GRADE guidelines: 1. Introduction-GRADE evidence profiles and summary of findings tables. J Clin Epidemiol 2011; 64: 383-394.

16 Review Manager (RevMan) version 5.3. Copenhagen, The Nordic Cochrane Centre, The Cochrane Collaboration; 2014.

17 Beasley R, Holliday M, Reddel HK, et al. Controlled trial of budesonide-formoterol as needed for mild asthma. N Engl J Med 2019; 380: 2020-2030.

18 Becker $\mathrm{AB}$, Kuznetsova $\mathrm{O}$, Vermeulen $\mathrm{J}$, et al. Linear growth in prepubertal asthmatic children treated with montelukast, beclomethasone, or placebo: a 56-week randomized double-blind study. Ann Allergy Asthma Immunol 2006; 96: 800-807.

19 Boulet LP, Turcotte H, Prince P, et al. Benefits of low-dose inhaled fluticasone on airway response and inflammation in mild asthma. Respir Med 2009; 103: 1554-1563.

20 den Otter JJ, van Schayck CP, Folgering HT, et al. Early intervention with inhaled corticosteroids in subjects with rapid decline in lung function and signs of bronchial hyperresponsiveness: results from the DIMCA programme. Eur J Gen Pract 2007; 13: 89-91.

21 Jonasson G, Carlsen KH, Jonasson C, et al. Low-dose inhaled budesonide once or twice daily for 27 months in children with mild asthma. Allergy 2000; 55: 740-748. 
22 Juniper EF, Kline PA, Vanzieleghem MA, et al. Effect of long-term treatment with an inhaled corticosteroid (budesonide) on airway hyperresponsiveness and clinical asthma in nonsteroid-dependent asthmatics. Am Rev Respir Dis 1990; 142: 832-836.

23 Merkus PJ, van Pelt W, van Houwelingen JC, et al. Inhaled corticosteroids and growth of airway function in asthmatic children. Eur Respir J 2004; 23: 861-868.

24 O'Byrne PM, FitzGerald JM, Bateman ED, et al. Inhaled combined budesonide-formoterol as needed in mild asthma. N Engl J Med 2018; 378: 1865-1876.

25 Osterman K, Carlholm M, Ekelund J, et al. Effect of 1 year daily treatment with 400 microg budesonide (Pulmicort Turbuhaler) in newly diagnosed asthmatics. Eur Respir J 1997; 10: 2210-2215.

26 Simons FE. A comparison of beclomethasone, salmeterol, and placebo in children with asthma. Canadian Beclomethasone Dipropionate-Salmeterol Xinafoate Study Group. N Engl J Med 1997; 337: 1659-1665.

27 Tonascia J, Adkinson NF, Bender B, et al. Long-term effects of budesonide or nedocromil in children with asthma. N Engl J Med 2000; 343: 1054-1063.

28 Agertoft L, Pedersen S. Effects of long-term treatment with an inhaled corticosteroid on growth and pulmonary function in asthmatic children. Respir Med 1994; 88: 373-381.

29 Backman H, Jansson SA, Stridsman C, et al. Chronic airway obstruction in a population-based adult asthma cohort: prevalence, incidence and prognostic factors. Respir Med 2018; 138: 115-122.

30 Bibi HS, Feigenbaum D, Hessen M, et al. Do current treatment protocols adequately prevent airway remodeling in children with mild intermittent asthma? Respir Med 2006; 100: 458-462.

31 Coumou H, Westerhof GA, De Nijs SB, et al. Predictors of accelerated decline in lung function in adult-onset asthma. Eur Respir J 2018; 51: 1701785.

32 Fujimura M, Nishizawa $\mathrm{Y}$, Nishitsuji $\mathrm{M}$, et al. Longitudinal decline in pulmonary function in atopic cough and cough variant asthma. Clin Exp Allergy 2003; 33: 588-594.

33 Grol MH, Gerritsen J, Vonk JM, et al. Risk factors for growth and decline of lung function in asthmatic individuals up to age 42 years: a 30-year follow-up study. Am J Respir Crit Care Med 1999; 160: 1830-1837.

34 Konig P, Shaffer J. The effect of drug therapy on long-term outcome of childhood asthma: a possible preview of the international guidelines. J Allergy Clin Immunol 1996; 98: 1103-1111.

35 Lange P, Scharling H, Ulrik CS, et al. Inhaled corticosteroids and decline of lung function in community residents with asthma. Thorax 2006; 61: 100-104.

36 Leung TF, Tang MF, Leung ASY, et al. Trajectory of spirometric and exhaled nitric oxide measurements in Chinese schoolchildren with asthma. Pediatr Allergy Immunol 2018; 29: 166-173.

37 Boulet LP, Jobin C, Milot J, et al. Five-year changes in airflow obstruction and airway responsiveness in mild to moderate asthma. Clin Invest Med 1994; 17: 432-442.

38 de Marco R, Marcon A, Jarvis D, et al. Inhaled steroids are associated with reduced lung function decline in subjects with asthma with elevated total IgE. J Allergy Clin Immunol 2007; 119: 611-617.

39 Adams NP, Bestall JC, Lasserson TJ, et al. Fluticasone versus placebo for chronic asthma in adults and children. Cochrane Database Syst Rev 2008: Cd003135.

40 Pauwels RA, Lofdahl CG, Postma DS, et al. Effect of inhaled formoterol and budesonide on exacerbations of asthma. Formoterol and Corticosteroids Establishing Therapy (FACET) International Study Group. $N$ Engl J Med 1997; 337: 1405-1411.

41 Santanello NC, Zhang J, Seidenberg B, et al. What are minimal important changes for asthma measures in a clinical trial? Eur Respir J 1999; 14: 23-27.

42 Yang CL, Simons E, Foty RG, et al. Misdiagnosis of asthma in schoolchildren. Pediatr Pulmonol 2017; 52: 293-302.

43 Aaron SD, Vandemheen KL, FitzGerald JM, et al. Reevaluation of diagnosis in adults with physician-diagnosed asthma. JAMA 2017; 317: 269-279.

44 Fu L, Freishtat RJ, Gordish-Dressman $\mathrm{H}$, et al. Natural progression of childhood asthma symptoms and strong influence of sex and puberty. Ann Am Thorac Soc 2014; 11: 939-944.

45 Agusti A, Hogg JC. Update on the pathogenesis of chronic obstructive pulmonary disease. N Engl J Med 2019; 381: $1248-1256$.

46 Bai TR, Vonk JM, Postma DS, et al. Severe exacerbations predict excess lung function decline in asthma. Eur Respir J 2007; 30: 452-456.

47 O’Byrne PM, Pedersen S, Lamm CJ, et al. Severe exacerbations and decline in lung function in asthma. Am J Respir Crit Care Med 2009; 179: 19-24.

48 Bateman ED, Reddel HK, O'Byrne PM, et al. As-needed budesonide-formoterol versus maintenance budesonide in mild asthma. N Engl J Med 2018; 378: 1877-1887.

49 Sterne JA, Hernan MA, Reeves BC, et al. ROBINS-I: a tool for assessing risk of bias in non-randomised studies of interventions. BMJ 2016; 355: i4919. 